\title{
Zolpidem Induced Sleep-related Eating and Complex Behaviors in a Patient with Obstructive Sleep Apnea and Restless Legs Syndrome
}

\author{
Young-Min Park, Hyun-Woo Shin \\ Department of Psychiatry, Ilsan Paik Hospital, Inje University College of Medicine, Goyang, Korea
}

\begin{abstract}
Zolpidem-induced sleep-related complex behaviors (SRCB) with anterograde amnesia have been reported. We describe herein a case in which the development of zolpidem-induced sleep-related eating disorder (SRED) and SRCB was strongly suspected. A 71-year-old Korean male was admitted to the Department of Psychiatry due to his repetitive SRED and SRCB with anterograde amnesia, which he reported as having occurred since taking zolpidem. The patient also had restless legs syndrome (RLS) and obstructive sleep apnea (OSA). His baseline serum iron level was low at admission. Zolpidem discontinuation resulted in the immediate disappearance of his SRED, but did not affect his RLS symptoms. These symptoms rapidly improved after adding a single i.v. iron injection once daily, and so he was discharged to day-clinic treatment. These findings indicate that zolpidem can induce SRCB. Although the pathophysiology of zolpidem-induced SRED and other SRCB remains unclear, clinicians should carefully monitor for the potential induction of complex behaviors associated with zolpidem in patients with comorbid RLS or OSA.
\end{abstract}

KEY WORDS: Zolpidem; Sleep-related eating disorder; Sleep-related complex behavior; Restless legs syndrome

\section{INTRODUCTION}

Zolpidem is an imidazopyridine hypnotic drug that has been world-widely prescribeed, since it reveals several clinical advantages such as short half-life and low abuse property comparative to benzodiazepine. ${ }^{1)}$ However, some severe adverse effects including sleep-related complex behaviors with anterograde amnesia have been reported. ${ }^{2)}$ Especially, sleep-related complex behaviors can be associated with accidents. ${ }^{2)}$ It included sleep eating, sleep driving, sleep cooking, and sleep conversation.

We describe herein a case report in which the development of zolpidem-induced sleep-related eating and complex behaviors was strongly suspected.

\section{CASE}

A 71-year-old male with 10-year history of hypertension, was admitted to the Department of Psychiatry in Ilsan Paik Hospital (Goyang, Korea) due to his repetitive

\footnotetext{
Received: July 21, 2015 / Revised: September 19, 2015

Accepted: September 23, 2015

Address for correspondence: Young-Min Park, MD, PhD Department of Psychiatry, IIsan Paik Hospital, Inje University College of Medicine, 170 Juhwa-ro, Ilsanseo-gu, Goyang 10380, Korea Tel: +82-31-910-7260, Fax: +82-31-910-7268 E-mail: medipark@hanmail.net
}

sleep-related eating and complex behaviors with anterograde amnesia. He reported that his repetitive sleep-related eating (once or twice a week) had occurred since zolpidem was added to previous prescription for 8 months. Several days ago, in the night time, he ate some food and went outside through the window and slept in his brother's house near his after receiving zolpidem. He was very perplexed at this event in next morning because he did not remember his behaviors at all. He had received zolpidem controlled release $12.5 \mathrm{mg}$, ativan $0.5 \mathrm{mg}$, and pramipexole $0.5 \mathrm{mg}$ bed time in another hospital where he recently had visited since his polysomnography revealed obstructive sleep apnea (OSA) and periodic limb movement. In spite of these medications, he still complained of insomnia and restless legs syndrome (RLS) symptoms including an uncomfortable sensation in both legs that worsened at rest or at night, and which could be relieved only by moving the legs. The patient met the diagnostic criteria for RLS published by the International Restless Legs Syndrome Study Group. ${ }^{3)}$ After admission, baseline serum iron level was $53 \mu \mathrm{g} / \mathrm{dl}$ (normal range, 60-180 $\mu \mathrm{g} / \mathrm{dl}$ ) although other laboratory finding and brain magnetic resonance imaging were all within a normal range. In addition, his cognitive function was within normal limit according to Mini-Mental State Examination Korean version. His sleep-related eating disappeared immediately

(c) This is an Open-Access article distributed under the terms of the Creative Commons Attribution Non-Commercial License (http://creativecommons.org/licenses/by-nc/4.0) which permits unrestricted non-commercial use, distribution, and reproduction in any medium, provided the original work is properly cited. 
after zolpidem discontinuation. He was initially treated with ferrous sulfate $256 \mathrm{mg}$ (oral iron), clonazepam 1.5 $\mathrm{mg}$, and ropinirole $1 \mathrm{mg}$ once daily at admission day 5 , but his insomnia and RLS symptoms did not resolve although his sleep-related eating disorder (SRED) disappeared. Thus, single intravenous (IV) iron (cosmofer $200 \mathrm{mg}$ IV) injection was added once in a day. After that, his RLS symptoms and insomnia rapidly improved, and his iron level increased to $234 \mu \mathrm{g} / \mathrm{dl}$. Thus, he was discharged to day-clinic treatment. Following discharge, he was maintained on a daily dose of 1 tablet of ferrous sulfate, $1 \mathrm{mg}$ of ropinirole and $1.5 \mathrm{mg}$ of clonazepam. Zolpidem administration had been discontinued after 9 months, and there was no recurrence of his complex behaviors.

\section{DISCUSSION}

Some investigators found that the prevalence of SRED was about $5 \%$ in general population. ${ }^{4)}$ Although there was no study that investigated the prevalence of zolpidem-induced SRED, reports related to this adverse effect of zolpidem increased more and more. ${ }^{5,6)}$ However, its pathophysiology has been unknown. SRED has been found to be associated with RLS in some studies. ${ }^{7)}$ Another studies found that it was associated with OSA. ${ }^{8)}$ Interestingly, our patient has both RLS and OSA. Schenck et al.$^{8)}$ observed that $80 \%$ of their SRED cohort have comorbid sleep disorder. In a recent study, comorbidities were frequent and included insomnia (58.8\%), RLS (47\%), sleep-disordered breathing (26\%), psychiatric disorders (38.2\%), and overweight or obesity (41.1\%). Sleep-related eating disappeared in most of zolpidem-induced SRED cases in most reports like our patient. ${ }^{9)}$ Thus, zolpidem is a potential cause of SRED. The hypothesis of this phenomenon seems to be related to the stimulating effect to brain of zolpidem. ${ }^{10)}$ Some investigators reported that zolpidem stimulated the patients' brain of vegetative state and caused them to awaken for short period. ${ }^{10,11)}$ In addition, some studies found that zolpidem improved the post-stroke Broca's aphasia, dystonia, blepharospasm, and quadriparesis although these results were preliminary. ${ }^{12)}$ In addition, zolpidem may induce parasomnias and increase non-rapid eye movement (non-REM) sleep. ${ }^{13,14)}$ So these properties increase risk of somnambulistic behaviors. ${ }^{15)}$ Another hypothesis is that zolpidem induce disinhibition or hallucinations with amnesia. Some patients presented their behaviors with anterograde amnesia after taking zolpidem, reporting hallucinatory experience before they fell asleep. ${ }^{15)}$ However, because in addition to zolpidem, an- other hypnotics (i.e., benzodiazepine) or psychotropics can induce SRED ${ }^{8,16,17)}$ further study related to the pathophysiology of SRED is needed in future.

Our patient presented complex behaviors including opening window, walking, visiting his brother's house, and sleeping as well as sleep eating. ${ }^{2)}$ All these behaviors disappeared immediately after the discontinuation of the zolpidem. In addition, zolpidem administration had been discontinued after 9 months, and there was no recurrence of his complex behaviors. Thus, this case is consistent with previous reports that zolpidem even can induce complex behaviors. Especially, RLS may produces fragmented sleep as well as the inability to stay in bed at awakenings. ${ }^{8}$ These partial arousals seem to eventually result in noturnal eating episodes. Furthermore, some investigators found that dopaminergic agents had some therapeutic benefit in patients with SRED and RLS. ${ }^{7)}$ In addition, the use of zolpidem seems to induce or aggaravate the presenting SRED because it has the stimulating effect to brain. ${ }^{10,11)}$ Thus, both of RLS and the use of zolpidem seem to be associated with the pathophysiology of SRED in our patient.

These complex behaviors included driving a car, cleaning the house, writing a e-mail with inputing correct user name and password to log on, engaging in conversation and sex. ${ }^{18,19)}$ Especially, these complex behaviors resulted in car accidents or crime such as violence and sex offense, ${ }^{2)}$ although cause and effect was not always clear.

Regarding to anterograde amnesia, our patient presented that most zolpidem-induced SRED and single zolpidem-induced complex behaviors were accompanied with the anterograde amnesia. ${ }^{9}$ Recent study also found that all except one patient (33/34) had partial or total amnesia of these events. ${ }^{5}$ Night eating syndrome (NES) is similar with SRED because this disorder also has eating problem in night-time. ${ }^{20)}$ Some investigators assumed that the difference between two disorders is the state of consciousness and amnesia during eating episode. ${ }^{9)}$ However, it is not easy to assess consciousness and amnesia during eating episode in night-time depending on patient's comment. Thus, it is also difficult to differentiate NES and SRED. ${ }^{20)}$

The treatment of SRED or sleep-related complex behaviors is not well established. However, it is important to consider the cause of SRED or sleep-related complex behaviors. ${ }^{8)}$ Most of all, the removal of zolpidem is central to the treatment. ${ }^{9}{ }^{9}$ In addition, underlying sleep disorder has to be treated like RLS and OSA. ${ }^{8)}$ In case of our patient, treatment was very complicated. He has RLS with iron deficiency, OSA, and zolpidem-induced SRED and 
complex behaviors. Thus, his treatment included RLS therapy with ropinirole, clonazepam, and iron as well as the discontinuation of zolpidem. Ropinirole and clonazepam have been maintained because his RLS symptoms had continued under normal iron level according to his past history.

In conclusion, zolpidem could induce sleep-related behaviors. Although the pathophysiology of zolpidem-induced SRED and other complex behaviors remains unclear, clinicians should carefully monitor for the potential induction of complex behaviors associated with zolpidem.

\section{Acknowledgments}

This study was supported by a grant from National Research Foundation of Korea (NRF), funded by Ministry of Education (NRF-2014R1A1A2A10059345).

\section{REFERENCES}

1. Fitzgerald AC, Wright BT, Heldt SA. The behavioral pharmacology of zolpidem: evidence for the functional significance of a 1-containing GABA(A) receptors. Psychopharmacology (Berl) 2014;231:1865-1896.

2. Daley C, McNiel DE, Binder RL. "I did what?" Zolpidem and the courts. J Am Acad Psychiatry Law 2011;39:535-542.

3. Allen RP, Picchietti D, Hening WA, Trenkwalder C, Walters AS, Montplaisi J; Restless Legs Syndrome Diagnosis and Epidemiology workshop at the National Institutes of Health; International Restless Legs Syndrome Study Group. Restless legs syndrome: diagnostic criteria, special considerations, and epidemiology. A report from the restless legs syndrome diagnosis and epidemiology workshop at the National Institutes of Health. Sleep Med 2003;4:101-119.

4. Winkelman JW, Herzog DB, Fava M. The prevalence of sleep-related eating disorder in psychiatric and nonpsychiatric populations. Psychol Med 1999;29:1461-1466.

5. Santin J, Mery V, Elso MJ, Retamal E, Torres C, Ivelic J, et al. Sleep-related eating disorder: a descriptive study in Chilean patients. Sleep Med 2014;15:163-167.

6. Nzwalo H, Ferreira L, Peralta R, Bentes C. Sleep-related eating disorder secondary to zolpidem. BMJ Case Rep 2013:2013:bcr2012008003. doi:10.1136/bcr-2012-008003.

7. Provini F, Albani F, Vetrugno R, Vignatelli L, Lombardi C, Plazzi G, et al. A pilot double-blind placebo-controlled trial of low-dose pramipexole in sleep-related eating disorder. Eur J Neurol 2005;12:432-436.

8. Schenck CH, Hurwitz TD, O'Connor KA, Mahowald MW. Additional categories of sleep-related eating disorders and the current status of treatment. Sleep 1993;16:457-466.

9. Auger RR. Sleep-related eating disorders. Psychiatry (Edgmont) 2006;3:64-70

10. Machado C, Estévez M, Rodríguez R, Pérez-Nellar J, Chinchilla M, DeFina $\mathrm{P}$, et al. Zolpidem arousing effect in persistent vegetative state patients: autonomic, EEG and behavioral assessment. Curr Pharm Des 2014;20:4185-4202.

11. Cohen SI, Duong TT. Increased arousal in a patient with anoxic brain injury after administration of zolpidem. Am J Phys Med Rehabil 2008;87:229-231.

12. Hoque R, Chesson AL Jr. Zolpidem-induced sleepwalking, sleep related eating disorder, and sleep-driving: fluorine18-flourodeoxyglucose positron emission tomography analysis. and a literature review of other unexpected clinical effects of zolpidem. J Clin Sleep Med 2009;5:471-476.

13. Yang W, Dollear M, Muthukrishnan SR. One rare side effect of zolpidem-sleepwalking: a case report. Arch Phys Med Rehabil 2005;86:1265-1266.

14. Feinberg I, Maloney T, Campbell IG. Effects of hypnotics on the sleep EEG of healthy young adults: new data and psychopharmacologic implications. J Psychiatr Res 2000; $34: 423-438$

15. Le Bon O, Neu D. Total absence of recall of discussion occurred shortly after ingestion of $10 \mathrm{mg}$ zolpidem. Pharmacopsychiatry 2009;42:126-127.

16. Paquet V, Strul J, Servais L, Pelc I, Fossion P. Sleep-related eating disorder induced by olanzapine. J Clin Psychiatry 2002;63:597.

17. Lu ML, Shen WW. Sleep-related eating disorder induced by risperidone. J Clin Psychiatry 2004;65:273-274.

18. Dolder CR, Nelson MH. Hypnosedative-induced complex behaviours : incidence, mechanisms and management. CNS Drugs 2008;22:1021-1036.

19. Siddiqui F, Osuna E, Chokroverty S. Writing emails as part of sleepwalking after increase in Zolpidem. Sleep Med 2009; 10:262-264.

20. Winkelman JW. Sleep-related eating disorder and night eating syndrome: sleep disorders, eating disorders, or both? Sleep 2006;29:876-877. 